\title{
Temperatur- und Lösungsmittelabhängigkeit der Breite von Relaxationszeitverteilungen starrer Dipolmoleküle *
}

\author{
Konrad Kreuter ** \\ Abteilung für Mikrowellenphysik am Physikalischen Institut der Universität Mainz
}

(Z. Naturforsch. 23 a, 1728-1740 [1968]; eingegangen am 23. Juli 1968)

\begin{abstract}
Das Relaxationsverhalten von starren Dipolmolekülen in verdünnter Lösung wird analysiert auf Grund von Absorptionsmessungen im Mikrowellengebiet mit Wellenlängen zwischen $33 \mathrm{~cm}$ und $2,3 \mathrm{~mm}$ bei einer Temperatur von $20^{\circ} \mathrm{C}$. Für die Dipolsubstanzen Benzophenon, 1-Bromnaphthalin, Chlorcyclohexan, Chlorbenzol und Tetrahydrofuran dienen als Lösungsmittel Heptan, Benzol, Methylcyclohexan, Dekalin und eine Mischung eines aliphatischen Öles mit Heptan. Die Abhängigkeit der Absorptionsgröße $\varepsilon^{\prime \prime}$ von der Frequenz, die Absorptionskurve, kann in keinem Fall durch die Debyesche Theorie mit einer einzigen Zeitkonstanten beschrieben werden. Alle Absorptionskurven sind breiter als die Debye-Kurve. Zur Interpolation der Meßwerte und zur Bestimmung der Breite der Absorptionskurve werden die von FröHLICH angegebenen Beziehungen benutzt, denen eine Relaxationszeitverteilung zugrunde liegt. Es wird gezeigt, daß im Rahmen der Meßgenauigkeit von ca. $2 \%$ keine Differenzierung möglich ist zwischen Absorptionskurven zu unterschiedlichen Verteilungsfunktionen für die Relaxationszeitverteilung, wie sie von verschiedenen Autoren angegeben werden (Cole und Cole ${ }^{3}$, Fuoss und Kirkwood ${ }^{4}$, W WGNer $^{7}$, Gevers ${ }^{5}$ und Fröhlich ${ }^{6}$ ). Es kann nur die Breite einer Relaxationszeitverteilung bestimmt werden; als Ma $\beta$ dafür dient ein Parameterwert $p=\ln \left(\tau_{1} / \tau_{2}\right)$. Die Ergebnisse der Analysen lassen erkennen, daß der Parameter $p$ stark abhängig ist vom Lösungsmittel und nur sehr wenig vom Dipolmolekül beeinflußt wird. $p$ wächst im allgemeinen mit steigender Viskosität der Lösung, doch kann diese nicht die bestimmende Größe sein. Die Messungen der Temperaturabhängigkeit von $p$ am Beispiel Chlorcyclohexan in Heptan (Temperaturbereich: $-40^{\circ} \mathrm{C}$ bis $\left.+40^{\circ} \mathrm{C}\right)$ und Dekalin $\left(-25^{\circ} \mathrm{C}\right.$ bis $\left.+90^{\circ} \mathrm{C}\right)$ lassen den Schluß zu, daß die Breite der beobachteten Relaxationszeitverteilung durch zwei Konstanten $-V_{0}$ und $T_{0}-$ und die Beziehung

$$
p=V_{0}\left(1-T / T_{0}\right) / R T
$$
\end{abstract}

beschrieben werden kann. $V_{0}$ entspricht einer Variationsbreite der Aktivierungsenergien von einigen $\mathrm{kcal} / \mathrm{Mol}, T_{0}$ ist eine charakteristische Temperatur, die im speziellen Fall gleich der Siedetemperatur der Lösung gesetzt werden kann.

Die Relaxation der elektrischen Dipolorientierung von starren Molekülen in der Flüssigkeit sollte nach der Debyeschen Theorie durch eine einzige Zeitkonstante beschrieben werden können. Charakteristisch dafür ist die bekannte Abhängigkeit der komplexen Dielektrizitätskonstanten $\varepsilon$ von der Frequenz, die Debyesche Beziehung

$$
\varepsilon(\omega)-\varepsilon_{\infty}=\left(\varepsilon_{0}-\varepsilon_{\infty}\right) /(1+i \omega \tau) .
$$

Je umfangreicher und genauer nun das Beobachtungsmaterial zum Relaxationsverhalten starrer $\mathrm{Di}$ polmoleküle im Laufe der letzten Jahre wurde, desto deutlicher zeigte sich, daß dieses nur allzu häufig nicht durch Gl. (1) zu beschreiben ist, sondern daß mehr oder minder starke Abweichungen zu beobachten sind ${ }^{1}$. Diese Erkenntnis ist freilich bisher nur bruchstückhaft, da sie meist im Rahmen von Un-

\footnotetext{
* D 77 - Ein Kurzvortrag wurde auf der Frühjahrstagung in Freudenstadt 1968 gehalten - Verhandl. DPG (VI) 3, 273 [1968].

* Adresse des Verfassers: INTERDIGIT, 7501 Wöschbach.
}

tersuchungen zu ganz anderen Fragestellungen nur nebenbei gewonnen wurde. Nur wenige Arbeiten sind speziell dem vom Debye-Charakter abweichenden Relaxationsverhalten starrer Dipolmoleküle gewidmet $^{2}$. Ein weiterer Schritt zur systematischen Erforschung dieses Phänomens soll daher mit der vorliegenden Arbeit getan sein. Bevor jedoch die Meßergebnisse im einzelnen diskutiert werden, ist $\mathrm{zu}$ überlegen, wie solche Meßergebnisse analysiert werden können, und was aus ihnen für das Relaxationsverhalten gefolgert werden kann.

\section{Analyse eines Relaxationsverhaltens}

Zur Analyse eines beobachteten Relaxationsverhaltens sind verschiedene Methoden entwickelt worden. Werden Real- und Imaginärteil der Dielektri-

1 Eine Fülle von Meßergebnissen ist von Sмyтн und Mitarbeitern in der Serie "Microwave Absorption and Molecular Structure in Liquids" veröffentlicht; siehe z. B. l. c. ${ }^{16}$ und l. c. ${ }^{17}$, außerdem auch 1. c. ${ }^{11}$.

2 W. Zeil, J. HaAse u. O. Stiefvater, Z. Elektrochemie 65 616 [1961]. - J. HaAse u. W. ZeIL, Z. Naturforsch. 18 a, 828 [1963] und auch l. c. ${ }^{20}$. 
zitätskonstanten $\varepsilon(\omega)=\varepsilon^{\prime}-i \varepsilon^{\prime \prime}$ in der komplexen Zahlenebene aufgetragen, dann entspricht der Beziehung (1) ein Halbkreis über der $\varepsilon^{\prime}$-Achse. Liegen die Meßwerte nicht auf einem solchen Halbkreis, so können sie nach CoLE und CoLE ${ }^{3}$ häufig durch einen Kreisbogen miteinander verbunden werden, dessen Mittelpunkt unterhalb der $\varepsilon^{\prime}$-Achse liegt. Als Parameter, der als Maß für die Abweichung vom DebyeCharakter benutzt wird, dient der Winkel

$$
\alpha=\pi / 2-2 \arctan \left(2 \varepsilon^{\prime \prime}{ }_{\max } / S\right)
$$

mit der Abkürzung $S=\varepsilon_{0}-\varepsilon_{\infty}$ für die Dispersionsstufe (vgl. Abb. 2). - Vor allem bei Untersuchungen an verdünnten Lösungen, auf die sich unsere Messungen beschränken, erlaubt die erforderliche Meßtechnik im Bereich der Mikrowellen meist nur eine Bestimmung von $\varepsilon^{\prime \prime}$ mit hinreichender Genauigkeit. Da andererseits die Frequenz als sehr genaue Meßgröße zur Verfügung steht, empfiehlt es sich, das Variablenpaar $\varepsilon^{\prime \prime}$ und $\omega$ zur Analyse heranzuziehen. In einem Diagramm, in dem $\operatorname{arcosh}\left(\varepsilon^{\prime \prime}{ }_{\max } / \varepsilon^{\prime \prime}(\omega)\right)$ über $\ln (\omega)$ aufgetragen ist, wird der Imaginärteil der Beziehung (1) durch eine Gerade mit der Steigung $\beta=1$ wiedergegeben. Nach Fuoss und Kinkwood ${ }^{4}$ können Meßwerte, die von dieser Geraden abweichen, durch andere Geraden interpoliert werden, deren Steigung $\beta<1$ ein Maß für die Abweichung vom Debye-Verhalten ist.

Beide soeben erwähnten Methoden erlauben eine einfache graphische Überprüfung des Relaxationsverhaltens mit Zirkel oder Lineal, denn dazu wurden sie wohl entwickelt. Dank der jederzeit mühelos einsetzbaren modernen elektronischen Rechenmaschinen ist man heute nicht mehr angewiesen auf solche einfachen graphischen Hilfsmittel. Man kann entweder dem Automaten eine rein rechnerische Auswertung der Ergebnisse übertragen, oder sich ebenso einfach für eine gewünschte Darstellungsweise geeignete Funktionsscharen berechnen lassen, um danach Schablonen anzufertigen. Wir haben deshalb unsere Meßergebnisse in einem Diagramm, in dem $\log \varepsilon^{\prime \prime}$ über $\log \omega$ aufgetragen ist, ausgewertet und wiedergegeben. Das hat verschiedene Vorteile, deren wichtigster wohl folgender ist: Die Abhängigkeit der Absorptionsgröße $\varepsilon^{\prime \prime}$ von der Frequenz - die Absorptionskurve - wird in einem solchen Diagramm durch einen Kurvenzug wiedergegeben, der von der Größe der zugehörigen Dispersionsstufe $S$

3 K. S. Cole u. R. H. Cole, J. Chem. Phys. 9, 341 [1941]. genau so unabhängig ist, wie von der absoluten Länge der entsprechenden Relaxationszeiten. Den aus Gl. (1) abgeleiteten Verlauf von $\varepsilon^{\prime \prime}(\omega)$ werden wir im folgenden als Debye-Kurve bezeichnen.

\section{Relaxationszeitverteilungen}

Ein Abweichen eines beobachteten Relaxationsverhaltens vom Debye-Charakter zeigt sich darin, daß die entsprechenden Meßpunkte nicht auf der Debye-Kurve liegen, sondern daß sie durch breitere Absorptionskurven miteinander verbunden werden müssen. Tritt eine gegenüber der Debye-Kurve verbreiterte Absorptionskurve auf, so kann dies durch die Annahme einer Relaxationszeitverteilung erklärt werden. Am allgemeinsten ist - vor allem bei der Untersuchung starrer Dipolmoleküle - der Ansatz einer kontinuierlichen Verteilung von Relaxationszeiten über ein begrenztes Intervall oder gar über das ganze Gebiet von 0 bis $\infty$. Für die Verteilungsfunktionen solcher kontinuierlicher Relaxationszeitverteilungen gibt es verschiedene Ansätze, die zum

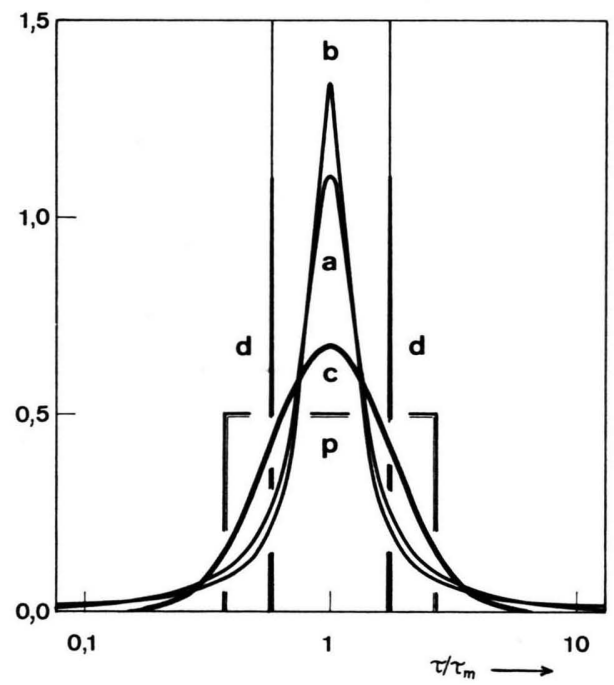

Abb. 1. Verteilungsfunktionen zur Beschreibung von Relaxationszeitverteilungen. Die Funktionen sind so normiert, daß

$\int^{+\infty} f(s) \mathrm{d} s=1$ mit $s=\ln \left(\tau / \tau_{\mathrm{m}}\right)$. (Zur Wahl der Parameterwerte vgl. Text.)

nach Cole und Cole nach Fuoss und Kirkwood nach Wagner

Parameter $\alpha=0,091$, Parameter $\beta=0,87$ rs bzw. Fröhlich zwei einzelne Relaxationszeiten nach Debye: $\tau_{1}=3 \tau_{2}$.

4 R. M. Fuoss u. J. G. Kirkwood, J. Am. Chem. Soc. 63, 385 [1941]. 
Teil aus analytischen Darstellungen von verbreiterten Absorptionskurven hergeleitet sind, zum Teil auch direkt aus molekülphysikalischen Modellen etwa im Zusammenhang mit der Platzwechseltheorie folgen. Abb. 1 zeigt einige der bekanntesten Verteilungsfunktionen.

Die einfachste Verteilungsfunktion, die von GEVERS $^{5}$ und in Verbindung mit Überlegungen zur Platzwechseltheorie von FröHLICH $^{6}$ vorgeschlagen wurde, läßt nur Relaxationszeiten zwischen zwei Grenzen $\tau_{1}$ und $\tau_{2}$ zu und lautet

$$
G(\tau) \mathrm{d} \tau=(1 / p) \cdot \mathrm{d} \tau / \tau .
$$

Der Parameter $p$ hängt von den Grenzen ab:

$$
p=\ln \left(\tau_{1} / \tau_{2}\right) ;
$$

er ist somit gleichzeitig ein anschauliches $\mathrm{Maß}$ für die Breite der Relaxationszeitverteilung. Nach FröHLICH kann der Parameter $p$ unmittelbar aus thermodynamischen Größen hergeleitet werden, worauf in der Diskussion noch näher eingegangen werden wird. - Von $\mathrm{W}_{\text {AGNER }}{ }^{7}$ stammt der Ansatz einer Wahrscheinlichkeitsverteilung der Relaxationszeiten - im logarithmischen Maßstab - um einen Schwerpunkt $\tau_{\mathrm{m}}$ mit $s=\ln \left(\tau / \tau_{\mathrm{m}}\right)$ :

$$
G(\tau) \mathrm{d} \tau=(c / \sqrt{ } \pi) \cdot \exp \left(-c^{2} s^{2}\right) \mathrm{d} s .
$$

Die beiden anderen in Abb. 1 noch gezeigten Verteilungsfunktionen sind aus den von Cole und Cole sowie von Fuoss und Kirkwood angegebenen Interpolationsfunktionen berechnet.

\section{Absorptionskurve und Verteilungsfunktion}

Der Verlauf der Absorptionskurve kann nicht kontinuierlich über den gesamten in Frage kommenden Frequenzbereich verfolgt werden, sondern nur für einige wenige Frequenzen gemessen werden. Zur Analyse des Relaxationsverhaltens müssen die Meßwerte daher mittels einer vorzugebenden Funktion $\varepsilon^{\prime \prime}(\omega)$ interpoliert werden, die zu jeder der in Abb. 1 gezeigten Verteilungsfunktionen angegeben werden kann. Es ist deshalb zu prüfen, wie stark oder wie schwach die Gestalt der Interpolationskurve von der speziellen Wahl der Verteilungsfunktion beeinflußt wird, oder auch umgekehrt, wie gut oder wie schlecht aus einem beobachteten Verlauf der Ab-

5 M. Gevers, Philips Res. Repts. 1, 197, 279, 361, 447 [1945/46].

${ }^{6}$ H. Fröhlich, Theory of Dielectrics, 2. Aufl., At the Clarendon Press, Oxford 1958. sorptionskurve auf eine zugehörige Verteilungsfunktion geschlossen werden kann (Vergleiche dazu auch die Überlegungen von StavermanN ${ }^{8}$ zur Meßbarkeit mechanischer Relaxationsspektren). Die in Abb. 1 zusammengestellten Relaxationszeitverteilungen sind in ihrer Gestalt so verschieden voneinander, daß ein Vergleich der entsprechenden Absorptionskurven eine ausreichende Prüfung erwarten läßt.

In Abb. 2 sind neben der schmalen spitzen DebyeKurve zwei verbreiterte Absorptionskurven eingezeichnet. Die eine davon gehört zu einer Verteilungsfunktion nach Cole und Cole mit einem Parameter-

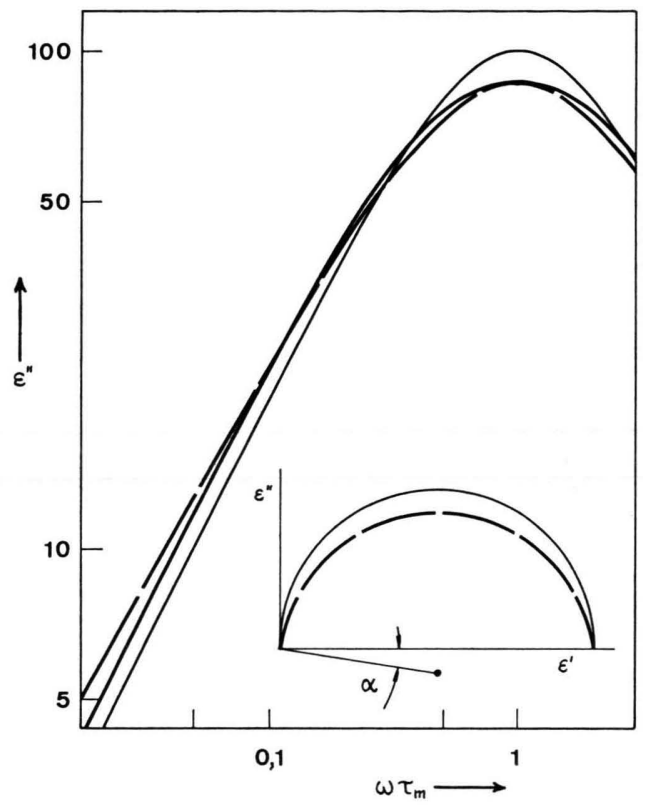

Abb. 2. Absorptionskurven für verschiedene Relaxationszeitverteilungen.

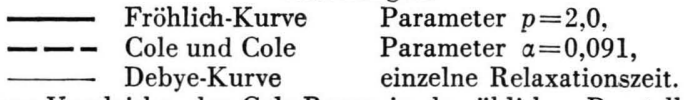

Zum Vergleich: der Cole-Bogen in der üblichen Darstellung.

wert $\alpha=0,091$, die andere zu einer Verteilungsfunktion nach Gevers bzw. Fröhlich mit entsprechendem Parameterwert $p=2,0$. Sie wird berechnet nach der von Fröhlich angegebenen analytischen Form

$$
\varepsilon^{\prime \prime}(\omega)=\frac{S}{p} \arctan \left(\frac{\sinh (p / 2)}{\cosh \left(\ln \omega \tau_{\mathrm{m}}\right)}\right)
$$

mit $S=\varepsilon_{0}-\varepsilon_{\infty}$ und $\tau_{\mathrm{m}}=\sqrt{\tau_{1} \tau_{2}}$ sowie Gl. (4). Dazu ist innerhalb der Zeichengenauigkeit eine Absorp-

${ }^{7}$ K. W. WAGner, Ann. Phys. Leipzig 40, 817 [1913] und Arch. Elektrotechn. 3, 83 [1914].

8 A. J. Stavermann, Kolloid-Z. 134, 197 [1953]. 
tionskurve nach Fuoss und Kirkwood mit $\beta=0,87$ ebensowenig von der Cole-Kurve zu unterscheiden, wie eine Absorptionskurve zur Verteilungsfunktion von Wagner mit $c=2,4-$ berechnet nach Y YGER ${ }^{9}$ von der Fröhlich-Kurve. Die Abweichungen der beiden gezeigten Kurven voneinander - und damit zugleich die Abweichungen der Absorptionskurven zu allen vier hier angeführten Verteilungsfunktionen mit entsprechenden Parameterwerten - sind auf beiden Seiten des Maximums der symmetrischen Kurven bis zu Werten von etwa einem Fünftel des Maximalwertes kleiner als - im ungünstigsten Fall ca. $5 \%$, wie die gewählte logarithmische Darstellung deutlich erkennen läßt. - Was bedeutet das für die Analyse von Meßergebnissen? Es liegen Meßpunkte vor, die irgendwie über einen solchen Kurvenverlauf verteilt sind. Bei einer Meßgenauigkeit von einigen Prozent - und das gilt für unsere Untersuchungen kann sicher nur unterschieden werden zwischen schmäleren und breiteren Absorptionskurven, nicht jedoch zwischen etwa gleichbreiten, aber zu verschiedenen Verteilungsfunktionen gehörenden Absorptionskurven, zumal wenn - wie das meist der Fall ist - die Meßwerte nur über einen begrenzten Frequenzbereich um das Maximum herum verteilt sind.

Noch deutlicher läßt sich die Unempfindlichkeit des Mittelstückes der Absorptionskurve gegenüber einer speziellen Verteilung der Relaxationszeiten durch folgendes belegen: Die Summenkurve, gebildet aus zwei Debye-Kurven gleicher Gewichte, deren Maxima um einen Faktor 3 auf der Frequenzskala gegeneinander verschoben sind, wäre in Abb. 2 ebenfalls nicht von der Fröhlich-Kurve zu unterscheiden. $\mathrm{Da}$ auch die geeignete Addition von mehr als zwei Debye-Kurven zu fast der gleichen Summenkurve führen kann, braucht daraufhin kaum noch erwähnt zu werden.

Solange Absorptionsmessungen nur mit einer Genauigkeit von einigen Prozent und innerhalb eines begrenzten Frequenzintervalles durchgeführt werden können, solange kann aus einer beobachteten symmetrischen Absorptionskurve, die breiter als die Debye-Kurve ist, nur auf die Breite - wiedergegeben durch einen Parameterwert - einer Relaxationszeitverteilung geschlossen werden, nicht jedoch auf eine spezielle Verteilungsfunktion, wenn nicht andere Kriterien dazu Anlaß geben könnten.

Bis zu einer Verbreiterung der Absorptionskurven von ca. $40 \%$ gegenüber einer Debye-Kurve - unsere späteren Analysen beschränken sich auf diesen

9 W. A. Y YGER, Physics 7, 434 [1936].
Bereich - können unter den genannten Voraussetzungen zwischen den unterschiedlichen Parametern der angeführten Verteilungsfunktionen die folgenden, empirisch durch Vergleich der Absorptionskurven gewonnenen Verknüpfungen benutzt werden:

$$
\begin{array}{ll}
\alpha \approx 0,0265 p^{1,8} & 0,7 \leqq p \leqq 3,0 \\
\beta \approx 1-1,45 \alpha & \\
c \approx 2,4 / p & 0,0 \leqq p \leqq 3,0 .
\end{array}
$$

\section{Fröhlich-Kurven}

Der Analyse eines beobachteten Relaxationsverhaltens muß, wie schon erwähnt, zuerst einmal eine spezielle Verteilungsfunktion zugrunde gelegt werden, da die wirklichen Absorptionskurven nicht über ihren ganzen Verlauf hinweg vermessen werden können, sondern nur an einigen wenigen Punkten, durch die dann eine Interpolationskurve gelegt werden muß. Wir wählen für diese Art der Auswertung die einfachste Relaxationszeitverteilung, die von Gevers bzw. Fröhlich vorgeschlagene, die außerdem gegenüber den drei anderen Funktionen den Vorzug hat, daß der zugehörige Parameter $p$ wie bereits angedeutet mit thermodynamischen Größen verknüpft werden kann.

Um abschätzen zu können, wie genau der Fröhlich-Parameter $p$ aus der vorgesehenen Analyse anzugeben sein wird, kann der Zusammenhang von $p$ mit der Halbwertsbreite der Fröhlich-Kurve benutzt werden. Ist $\omega_{\mathrm{h}}$ die Frequenz, für die entsprechend $\varepsilon^{\prime \prime}\left(\omega_{\mathrm{h}}\right)=0,5 \varepsilon^{\prime \prime}\left(\omega_{\max }\right)$ die Absorption nur noch die Hälfte des Maximalwertes beträgt, so kann zwischen der Halbwertsbreite $b=2\left|\ln \left(\omega_{\max } / \omega_{\mathrm{h}}\right)\right|$ und $p$ durch elementare Umformungen aus (6) die einfache Beziehung

$$
\cosh (b / 2)=1+\cosh (p / 2)
$$

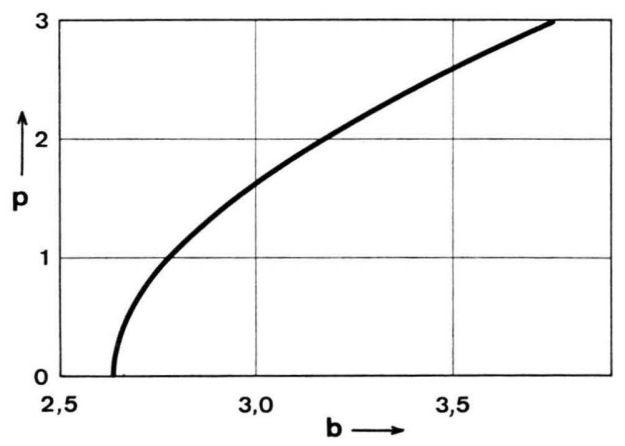

Abb. 3. Zusammenhang zwischen dem Parameter $p$ und der Breite $b$ einer Fröhlich-Kurve: $\cosh (b / 2)=1+\cosh (p / 2) \quad$ mit $\quad b=2 \mid \ln \left(\omega_{\max } / \omega_{\mathrm{h}}\right)$. 
hergeleitet werden. Abb. 3 zeigt den Zusammenhang zwischen $p$ und $b$ in dem für unsere Analysen wichtigen Bereich. Die Halbwertsbreite selbst kann in erster Näherung etwa genauso genau angegeben werden, wie die Absorptionsmessungen selbst sind. Das bedeutet nun aber bei einer Meßgenauigkeit von einigen Prozent, daß solche Absorptionskurven, denen ein $p$ von 0,5 oder noch weniger zukommt, nur sehr schwer wenn überhaupt von einer Debye-Kurve zu unterscheiden sind. Anders ausgedrückt heißt das, daß selbst dann, wenn innerhalb der Fehlergrenzen eine Debye-Kurve vorzuliegen scheint, statt einer einzigen Relaxationszeit sehr wohl auch eine Relaxationszeitverteilung vorhanden sein kann, deren Breite immerhin einem Verhältnis der Grenzen von $\tau_{1} / \tau_{2}=1,5$ entsprechen kann!

\section{Apparaturen und Substanzen}

Zur Untersuchung der Breite einer Relaxationszeitverteilung ist ein experimenteller Aufwand erforderlich, der weit über den hinaus geht, der etwa zur Bestimmung der mittleren Relaxationszeit allein nötig ist. Dafür genügt oft schon ein einziger Mikrowellenmeßpunkt, auf alle Fälle deren zwei, wenn die Dispersionsstufe entsprechend der "optischen “ Methode zur Bestimmung des Dipolmomentes durch $\varepsilon_{0}-n_{\mathrm{D}}{ }^{2}$ approximiert wird. Um die Breite einer Absorptionskurve - und damit die Breite einer Relaxationszeitverteilung - vor allem auch frei von nähernden Annahmen über die Größe von $S$ sicher bestimmen zu können, müssen mehrere Mikrowellenmeßpunkte in der Umgebung des Maximums einen Frequenzbereich von etwa zwei Dekaden überdecken!

\section{Mikrowellenapparaturen}

Uns standen betriebsbreite Anlagen zur Verfügung, mit denen $\varepsilon^{\prime \prime}(\omega)$ für acht verschiedene Frequenzen zwischen $1 \mathrm{GHz}$ und $100 \mathrm{GHz}$ gemessen werden kann. Dieses Spektrum von Meßfrequenzen ermöglicht es Relaxationszeitverteilungen zu erfassen, deren Schwerpunkte $\tau_{\mathrm{m}}$ im Bereich zwischen etwa $4 \mathrm{ps}$ und $50 \mathrm{ps}$ liegen. Da es gebräuchlich ist, in diesem Spektralbereich statt der Frequenz die Wellenlänge im Vakuum als Maßzahl zu benutzen, wird im folgenden die entsprechende Wellenlänge angegeben werden.

Die verwendeten Apparaturen wurden entwickelt zur Untersuchung schwach dämpfender Flüssigkeiten, wozu ja gerade die sogen. verdünnten Lösungen zählen. Die

10 G. Klages u. R. Langpape, Z. Naturforsch. 15 a, 964 [1960].
Absorptionswerte für Wellen mit $33,4 \mathrm{~cm}, 16,7 \mathrm{~cm}$ und $11,1 \mathrm{~cm}$ Wellenlänge werden aus der Halbwertsbreite der Resonanzkurve eines Koaxialresonators berechnet. Die Apparaturen für die kürzeren Wellen haben als Wellenleiter und Meßzellen Hohlrohre. Nach dem Prinzip eines Hohlrohr-Interferometers arbeitet die Anlage für 3,02 cm-Wellen. Die Differenz der Durchlaßkoeffizienten einer Meßzelle konstanter Länge, die einmal mit dem reinen Lösungsmittel, dann mit der zu untersuchenden Lösung gefüllt ist, ermöglicht die Berechnung der Absorption von 1,50 cm-Wellen. Die Änderung der empfangenen Leistung mit der Länge einer durchstrahlten Flüssigkeitssäule im Hohlrohr ist das Dämpfungsmaß für die $7,01 \mathrm{~mm}$-Wellen, ebenso für die 4,0 mm-Wellen. Nach dem Interferometerprinzip, jedoch mit Wellenbündeln im freien Raum, wird die Absorption der 2,3 mm-Wellen gemessen.

\section{Dipolmoleküle und Lösungsmittel}

Die untersuchten Substanzen wurden nach folgenden Gesichtspunkten ausgewählt: Die Dipolmoleküle müssen starr sein, damit eine innere Beweglichkeit als Ursache von verbreiterten Absorptionskurven ausgeschlossen werden kann. Außerdem dürfen die Moleküle untereinander nicht zur Assoziation neigen, denn die Bildung von Assoziationskomplexen kann ebenfalls Ursache verbreiterter Absorptionskurven sein. Schließlich sollten sich die Dipolmoleküle und auch die Lösungsmittel untereinander möglichst weitgehend in den Parametern unterscheiden, die auch die Relaxationszeit selbst bzw. $\tau_{\mathrm{m}}$ als Schwerpunkt einer Verteilung beeinflussen. Denn es war zu hoffen oder sogar zu vermuten, $\mathrm{da} \beta$ diese Parameter auch die Breite einer Verteilung beeinflussen.

Für die Dipolmoleküle sind solche Parameter vor allem das Molekülvolumen - besonders bei sehr kleinen Molekülen wurden schon vor Jahren Relaxationszeitverteilungen vermutet ${ }^{10}$ - und der von EIchноF und Hufnager ${ }^{11}$ angegebene effektive Dipolradius. Leider haben sehr kleine Dipolmoleküle auch sehr kurze Relaxationszeiten, so daß die Maxima der Absorptionskurven an den kurzwelligen Rand des uns zur Verfügung stehenden Frequenzbereiches rutschen. Eine Messung der Halbwertsbreite der Absorptionskurve wird dann unmöglich. Sehr große Moleküle sind oft nicht mehr starr genug oder lassen sich nicht in der erforderlichen Konzentration lösen, abgesehen davon, daß ihr Absorptionsmaximum an den langwelligen Rand des Frequenzbereiches herankommt. Der Variation des Volumens der Dipolmoleküle sind somit deutliche Grenzen gesetzt. Wir wählten als ersten Satz von Dipolsubstanzen: Benzophenon, 1-Bromnaphthalin, Chlorcyclohexan, Chlorbenzol und Tetrahydrofuran.

Für die Auswahl der Lösungsmittel kommt als entscheidender Parameter neben dem Molekülvolumen vor allem die Viskosität in Betracht, die zumindest bei

11 U. Eichhof f u. F. Hufnagel, Z. Naturforsch. 20 a, 630 [1965]. 
größeren Dipolmolekülen die Relaxationszeit entscheidend beeinflußt. Deshalb wurde außer Heptan, Benzol, Methylcyclohexan und Dekalin - in der Form des handelsüblichen Gemisches aus ca. 2 Teilen cis- und 1 Teil trans-Dekahydronaphthalin - eine Mischung, bestehend aus einem Öl langkettiger Aliphate* und aus Heptan im Volumenverhältnis $4: 1$, mit einer Viskosität von $7,0 \mathrm{cP}$ bei $20^{\circ} \mathrm{C}$ als Lösungsmittel benutzt.

\section{Meßergebnisse und Analysen}

Um einen ersten Überblick über das Relaxationsverhalten der ausgewählten Dipolmoleküle zu gewinnen, wurde jede Dipolsubstanz mit jedem Lösungsmittel kombiniert, und die Absorptionswerte $\varepsilon^{\prime \prime}(\omega)$ der verdünnten Lösungen für mindestens fünf der acht möglichen Frequenzen bei einer Temperatur von $20^{\circ} \mathrm{C}$ von jeweils fünf verschiedenen Konzentrationen gemessen. Die Ergebnisse wurden auf eine Konzentration von einheitlich $10^{20}$ Dipolen pro $\mathrm{cm}^{3}$ Lösung bezogen der Analyse unterworfen. Die Ergebnisse der Messungen sowie der Analysen zeigen die Abb. 4.1 bis 4.5 und Tab. 1.

Wie die Abbildungen erkennen lassen, gelingt eine Interpolation der Meßergebnisse mittels der von Fröhlich angegebenen Beziehung (6) sehr gut. Alle Absorptionskurven sind breiter als eine DebyeKurve. Als Maß für die Abweichung vom DebyeCharakter dient der Parameter $p$ in Tab. 1. Natürlich soll damit in keiner Weise eine Einschränkung vorgenommen worden sein derart, daß eine Relaxationszeitverteilung nach Fröhlich vorliegen muß und andere Verteilungsfunktionen ausgeschlossen wären. $p$ ist lediglich das Maß für eine nicht unbedingt als scharfe Grenze anzusehende Breite der Relaxationszeitverteilung.

Die Ungenauigkeit der Absorptionsmessungen von ca. $2 \%$ bringt es mit sich, daß die Parameterwerte erst von $p=1$ an auf höchstens $\pm 0,1$ sicher angegeben werden können - vgl. Abb. 3. Ein $p=0,7$ heißt deshalb in Tab. 1 nur, daß die Absorptionskurve unter Berücksichtigung der Meßfehler gerade als etwas breiter zu erkennen sind, als es eine Debye-Kurve sein müßte. Im Rahmen dieser Einschränkungen ist auf Grund der Zahlen von Tab. 1 die Breite der vorliegenden Relaxationszeitverteilungen für alle untersuchten Dipolmoleküle

* Weißöl W 505 der Fa. Rudolf Fuchs, Mineralölwerk Mannheim.

12 W. Jackson u. J. G. Powles (Trans. Faraday Soc. 42 a, 101 [1946]) geben für verdünnte Lösung von Benzophenon in nahezu gleich, obwohl sich die Volumina stark voneinander unterscheiden. Sie hängt nur ab vom Lösungsmittel. Das ist ein überraschendes Ergebnis, da man bisher der Ansicht war, daß zumindest große Moleküle, wie zum Beispiel das Bromnaphthalin, eine einzige Relaxationszeit zeigen, und daß nur bei kleinen Molekülen die Struktur der Umgebung eine Relaxationszeitverteilung hervorruft.

Die Absorptionskurven für Benzophenon in den hochviskosen Lösungsmitteln ${ }^{12}$ - Dekalin und aliphatische Mischung - lassen sich als einzige unter den beobachteten nicht durch einzelne Fröhlich-Kurven nähern. Die Flanken zu kürzeren Wellenlängen hin sind flacher als in den übrigen Fällen. Dieses Verhalten könnte auf eine unsymmetrische Relaxationszeitverteilung hindeuten. Zur weiteren Klärung sind zusätzliche Messungen im Millimetergebiet vorgesehen.

Alle Absorptionskurven des Tetrahydrofurans haben ihre Maxima erst bei Wellenlängen von weniger als $7 \mathrm{~mm}$. Das verfügbare Mikrowellenspektrum reicht deshalb nicht aus, die Halbwertsbreite und damit auch $p$ auf Grund von Absorptionsmessungen allein zu bestimmen. Jedoch widersprechen die Meßergebnisse nicht der Annahme, daß die $p$-Werte für das Tetrahydrofuran denen der übrigen vier Dipolsubstanzen gleich sind. Aus dem Anstieg der Flanken kann allerdings sicher geschlossen werden, daß ein vorliegende Verteilungsfunktion auf der Seite großer $\tau$-Werte eher den Charakter einer Verteilung nach Fröhlich oder Wagner hat, als den einer Verteilung nach Cole-Cole oder Fuoss-Kirkwood. Sonst müßten - vgl. Abb. 2 - weitab vom Maximum doch Abweichungen der Meßpunkte von der Fröhlich-Kurve zu beobachten sein.

\section{Dispersionsstufe}

Der dritte, neben $\tau_{\mathrm{m}}$ und $p$ für die Absorptionskurve nach Gl. (6) bestimmende Parameter, die Dispersionsstufe $S$, kann verglichen werden mit Messungen der Differenzen $\varepsilon_{0}-n_{\mathrm{D}}{ }^{2}$, die entsprechend der "optischen“ Methode zur Bestimmung des Dipolmomentes aus verdünnter Lösung häufig als Näherung für $S$ benutzt wird.

Bei den Dipolmolekülen Benzophenon, Chlorcyclohexan und Chlorbenzol stimmen innerhalb der Feh-

Paraffin (Viskosität $197 \mathrm{cP}$ bei $19^{\circ} \mathrm{C}$ ) ein $\beta=0,68$ nach Fuoss und Kirkwood als Parameter für die beobachtete verbreiterte Absorptionskurve an. Das entspricht einem $p$ von ca. 3,5 nach $\mathrm{Gl}$. (7). 


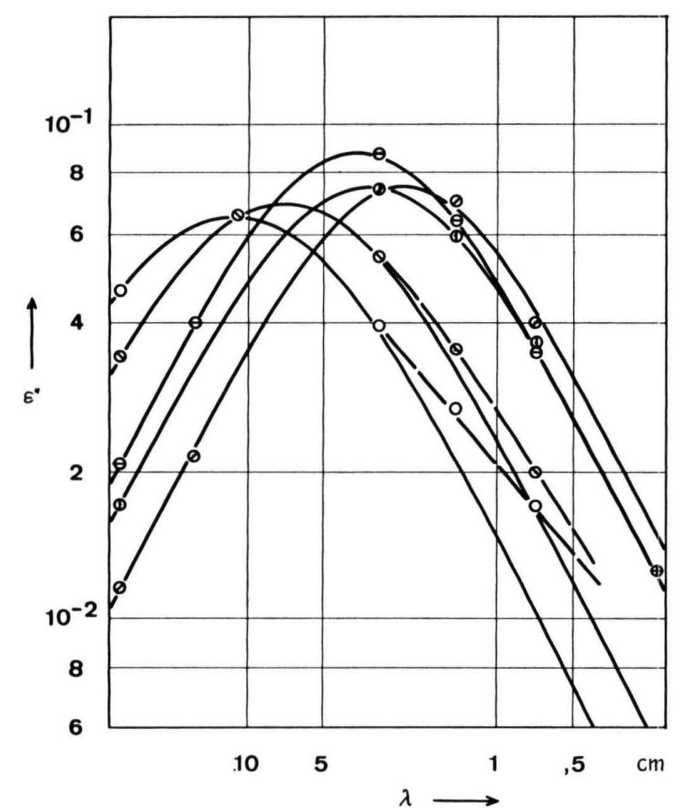

Abb. 4.1. Benzophenon.

$\mathrm{Zu} \mathrm{Abb.} \mathrm{4.1} \mathrm{bis} \mathrm{4.5.} \mathrm{Absorptionskurven} \mathrm{starrer} \mathrm{Di-}$ polmoleküle in verdünnter Lösung. Absorptionswerte $\varepsilon^{\prime \prime}$ für Konzentrationen von $10^{20}$ Dipolen pro $\mathrm{cm}^{3}$ Lösung. Interpolationskurven nach Fröhlich Gl. (6). Kennzeichnung der Lösungsmittel: $\oslash$ Heptan, $\ominus$ Benzol, (1) Methylcyclohexan, $\odot$ Dekalin, aliphat. Mischung. Temperatur $20^{\circ} \mathrm{C}$. Analysedaten in Tab. 1.
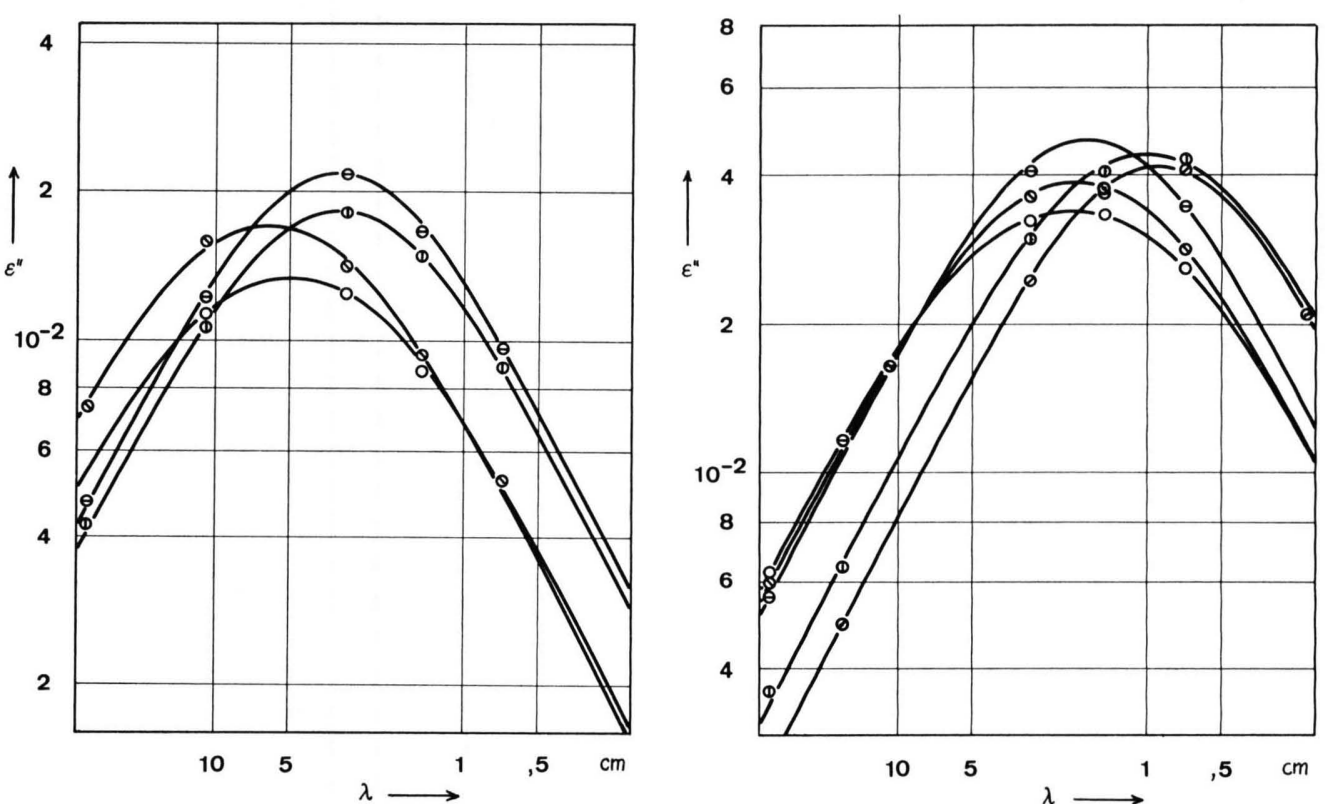

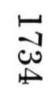

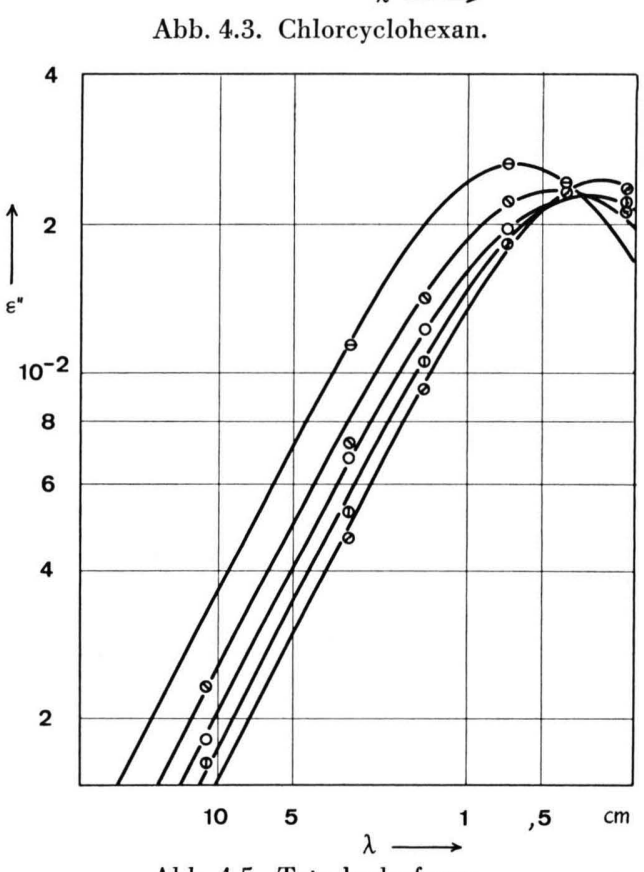

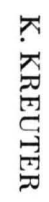

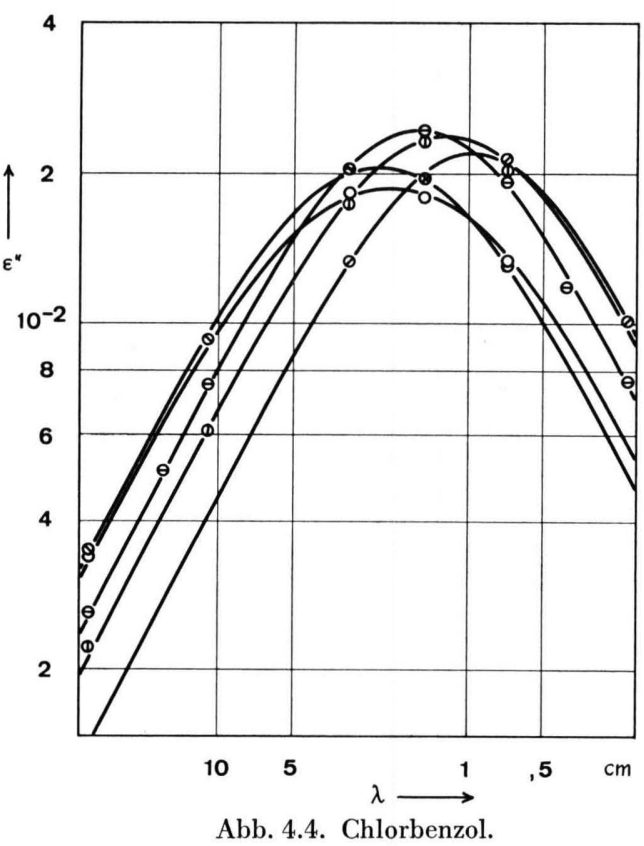

Abb. 4.5. Tetrahydrofuran 


\begin{tabular}{|c|c|c|c|c|c|}
\hline & $\frac{\pi}{0}$ & 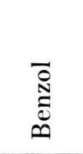 & 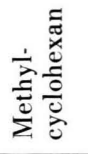 & 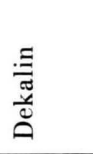 & 递 \\
\hline \multicolumn{6}{|c|}{ Benzophenon } \\
\hline $\begin{array}{c}\tau_{\mathrm{m}}[\mathrm{ps}] \\
\boldsymbol{p} \\
100^{\circ} \cdot S\end{array}$ & $\begin{array}{r}12,5 \\
\mathbf{0 , 7} \\
15,4\end{array}$ & $\begin{array}{r}19,1 \\
1,0 \\
18,4\end{array}$ & $\begin{array}{r}17,0 \\
1,4 \\
16,2\end{array}$ & $\begin{array}{l}38 \\
(\mathbf{1}, \mathbf{6}) \\
17\end{array}$ & $\begin{array}{l}60 \\
(\mathbf{1}, \mathbf{8}) \\
16\end{array}$ \\
\hline \multicolumn{6}{|c|}{ l-Bromnaphthalin } \\
\hline $\begin{array}{c}\tau_{\mathrm{m}}[\mathrm{ps}] \\
\boldsymbol{p} \\
100 \cdot S\end{array}$ & & $\begin{array}{c}17,0 \\
\mathbf{1 , 0} \\
4,55\end{array}$ & $\begin{array}{c}16,8 \\
\mathbf{1 , 4} \\
3,95\end{array}$ & $\begin{array}{l}32 \\
\mathbf{1 , 8} \\
3,85\end{array}$ & $\begin{array}{l}25 \\
\mathbf{2 , 2} \\
3,20\end{array}$ \\
\hline \multicolumn{6}{|c|}{ Chlorcyclohexan } \\
\hline $\begin{array}{c}\tau_{\mathrm{m}}[\mathrm{ps}] \\
\underset{100}{\boldsymbol{P}} \cdot S\end{array}$ & $\begin{array}{l}4,8 \\
1,0 \\
8,7\end{array}$ & $\begin{array}{l}9,1 \\
1,2 \\
9,8\end{array}$ & $\begin{array}{l}5,3 \\
1,6 \\
9,7\end{array}$ & $\begin{array}{r}10,2 \\
\mathbf{1 , 8} \\
8,6\end{array}$ & $\begin{array}{r}10,5 \\
2,4 \\
8,1\end{array}$ \\
\hline \multicolumn{6}{|c|}{ Chlorbenzol } \\
\hline $\begin{array}{c}\tau_{\mathrm{m}}[\mathrm{ps}] \\
\underset{p}{\boldsymbol{p}} \\
100 \cdot S\end{array}$ & $\begin{array}{l}5,2 \\
\mathbf{0 , 7} \\
4,45\end{array}$ & $\begin{array}{l}8,2 \\
1,0 \\
5,10\end{array}$ & $\begin{array}{l}6,5 \\
1,4 \\
5,10\end{array}$ & $\begin{array}{l}12,0 \\
1,6 \\
4,50\end{array}$ & $\begin{array}{c}11,1 \\
\mathbf{2 , 2} \\
4,40\end{array}$ \\
\hline \multicolumn{6}{|c|}{ Tetrahydrofuran } \\
\hline $\begin{array}{c}\tau_{\mathrm{m}}[\mathrm{ps}] \\
\boldsymbol{p} \\
100 \cdot S\end{array}$ & $\begin{array}{l}1,5 \\
\mathbf{0 , 7} \\
5,0\end{array}$ & $\begin{array}{l}3,3 \\
\mathbf{1 , 0} \\
5,5\end{array}$ & $\begin{array}{l}1,7 \\
1,4 \\
4,8\end{array}$ & $\begin{array}{l}2,3 \\
\mathbf{1 , 6} \\
5,2\end{array}$ & $\begin{array}{l}1,9 \\
2,0 \\
5,0\end{array}$ \\
\hline
\end{tabular}

Tab. 1. Analyse nach Fröhlich Gl. (6) der Absorptionsmessungen an verdünnten Lösungen - (Abb. 4.1 bis 4.5) -

Dispersionsstufe $S$ für $10^{20}$ Dipole pro $\mathrm{cm}^{3}$ Lösung. Tetrahydrofuran: siehe Text.

lergrenzen die Werte für die Dispersionsstufe, einmal gemessen nach der optischen Methode, zum anderen bestimmt aus den Absorptionsdaten allein, überein, wenn als Lösungsmittel die niederviskosen Verbindungen Heptan, Benzol und Methylcyclohexan verwendet werden. Deutliche Abweichungen bis zu $10 \%$ treten hingegen auf für die Lösungen mit Dekalin und der aliphatischen Mischung. Ähnlich ist es auch beim 1-Bromnaphthalin, doch sind da wegen der Unsicherheit hinsichtlich des Ultrarotgliedes keine so präzisen Aussagen möglich. Tetrahydrofuran kann zu diesen Vergleichen nicht herangezogen werden, da die Absorptionskurven nur gerade bis zum Maximum vermessen werden können, und $S$ nur auf Grund der Annahmen über den Parameter $p$ berechnet werden kann. Die in Tab. 1 angegebenen $p$-Werte führen allerdings zu Werten für $S$, die generell ca. $10 \%$ kleiner sind als die entsprechenden Werte $\varepsilon_{0}-n_{\mathrm{D}}{ }^{2}$, auch für die niederviskosen Lösungen. Es könnte deshalb sein, daß die Absorptions-

13 O. F. Kalman u. C. P. Smyth, J. Am. Chem. Soc. 82, 783 [1960]. kurven unsymmetrisch sind, d. h. die Flanken zu kürzeren Wellenlängen hin flacher abfallen als zu langen Wellen. Eine Klärung dieser Frage ist aber erst durch Absorptionsmessungen im Submillimetergebiet zu erwarten.

Unterschiede zwischen den aus den Absorptionsmessungen gewonnenen Werten für $S$ und $\varepsilon_{0}-n_{\mathrm{D}}{ }^{2}$ wurden auch von anderen Autoren beobachtet. So berichten Kalman und Smyth ${ }^{13}$ sowie DiCarlo und Sмутн ${ }^{14}$ von bis zu $10 \%$ kleineren Werten $S$ gegenüber $\varepsilon_{0}-n_{\mathrm{D}}{ }^{2}$, die aus Analysen nach Cole und Cole von Untersuchungen an starren Dipolmolekülen in hochviskosen Lösungsmitteln (Dekalin und Nujol) gewonnen wurden. Auch PoLey ${ }^{15}$ findet bei den Analysen (ebenfalls nach Cole und Cole) seiner Messungen an reinen Dipolstoffen Unterschiede zwischen $\varepsilon_{\infty}$ und $n_{\mathrm{D}}{ }^{2}$.

Da die erwähnten Abweichungen experimentell gesichert nur bei den Messungen mit hochviskosen Lösungsmitteln zu beobachten sind, könnte eine Erklärung darin zu suchen sein, daß die tatsächliche Relaxationszeitverteilung nicht der symmetrischen, der Auswertung zugrunde gelegten Verteilungsfunktion entspricht. Es wäre eine asymmetrische Verteilung denkbar, die eine ebensolche Absorptionskurve zur Folge hätte. Doch könnte eine solche Asymmetrie u. U. der nur auf einzelne Meßwerte innerhalb eines begrenzten Frequenzbereiches beschränkten Beobachtung verborgen geblieben sein.

\section{Temperaturabhängigkeit der Verteilungsbreite}

Neben der Abhängigkeit der Breite einer Relaxationszeitverteilung vom Lösungsmittel interessiert vor allem deren Temperaturabhängigkeit. Diese wurde am Beispiel verdünnter Lösungen von Chlorcyclohexan in Heptan und Dekalin untersucht. Beide Lösungsmittel sind in einem relativ großen und dem Experiment recht gut zugänglichen Temperaturbereich flüssig. Die Ergebnisse dieser Untersuchungen sind in Abb. 5.1 und 5.2 sowie in Tab. 2 zusammengefaßt.

Auf den ersten Blick zu erkennen ist die deutliche Abnahme der Verteilungsbreite mit zunehmender Temperatur. In der Diskussion wird dies noch ausführlich erörtert. Eine solche Abhängigkeit zwischen einem Verteilungsparameter und der Temperatur ist

\footnotetext{
14 E. N. DiCarlo u. C. P. Smyth, J. Phys. Chem. 66, 1105 [1962].

15 J. Ph. Poley, Appl. Sci. Res. Sect. B 4, 337 [1955].
} 


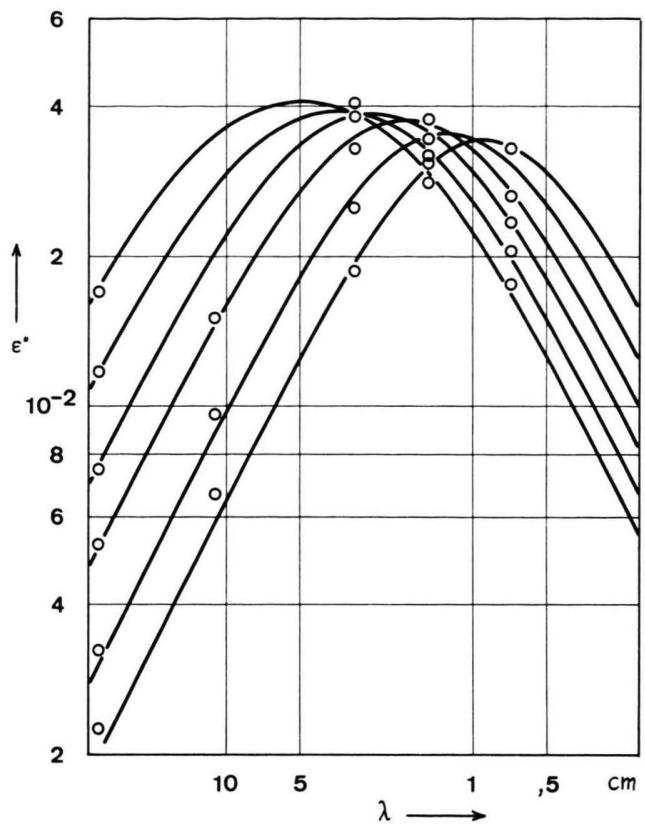

Abb. 5.1. Lösungsmittel: Dekalin.

Dipolmolekül: Ch lor c y c l o h ex a n

Lösungsmittel: D e k a l in

$\begin{array}{crrrrrr}\text { Temp. }\left[{ }^{\circ} \mathrm{C}\right] & -25^{\circ} & -10^{\circ} & +10^{\circ} & +30^{\circ} & +60^{\circ} & +90^{\circ} \\ \tau_{\mathrm{m}}[\mathrm{ps}] & 25,8 & 19,0 & 13,4 & 9,8 & 6,7 & 4,8 \\ \boldsymbol{p} & \mathbf{2 , 5} & \mathbf{2 , 1} & \mathbf{1 , 8} & \mathbf{1 , 5} & \mathbf{1 , 2} & \mathbf{0 , 7} \\ 100 \cdot S & 9,9 & 9,4 & 8,8 & \mathbf{8 , 2} & 7,5 & 6,9\end{array}$

Lösungsmittel: Hep t a n

\begin{tabular}{|c|c|c|c|c|}
\hline Temp. $\left[{ }^{\circ} \mathrm{C}\right]$ & $-40^{\circ}$ & $-20^{\circ}$ & $+10^{\circ}$ & $+40^{\circ}$ \\
\hline$\tau_{\mathrm{m}}[\mathrm{ps}]$ & 9,7 & 7,2 & 5,1 & 4,1 \\
\hline $\boldsymbol{p}$ & 2,0 & 1,6 & 1,1 & 0,7 \\
\hline $100 \cdot S$ & 12,0 & 10,7 & 9,3 & 8,1 \\
\hline
\end{tabular}

schon früher beobachtet worden, allerdings meist im Rahmen von Messungen zu anderen Fragestellungen und für ein viel kleineres Temperaturintervall.

Verdünnte Lösungen von 1-Bromnaphthalin wurden auch von GrubB und Sмyth ${ }^{16}$ untersucht. Als Lösungsmittel dienten Dekalin und Nujol, letzteres eine äußerst viskose Flüssigkeit $(\eta=211 \mathrm{cP}$ bei $20{ }^{\circ} \mathrm{C}$ ). Ihre Analysen nach Cole und Cole können sich auf fünf Meßwerte im Wellenlängenbereich zwischen $50 \mathrm{~cm}$ und $1,25 \mathrm{~cm}$ stützen. Wertet man die in Zahlen angegebenen Absorptionswerte nach der von uns angewandten Methode aus, dann sind die etwas streuenden Meßwerte am besten durch Fröhlich-Kurven zu interpolieren, denen Verteilungsbrei-

16 E. L. Grubb u. C. P. Sмyth, J. Am. Chem. Soc. 83, 4122 [1961].

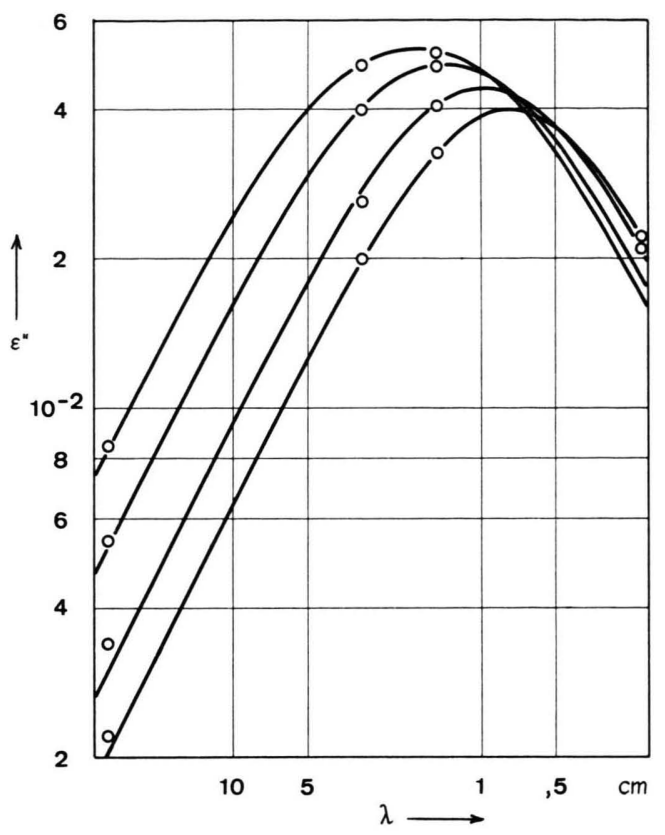

Abb. 5.2. Lösungsmittel: Heptan.

$\mathrm{Zu} \mathrm{Abb.} 5.1$ und 5.2. Absorptionsverhalten von Chlorcyclohexan in verdünnter Lösung in Abhängigkeit von der Temperatur. Absorptionswerte $\varepsilon^{\prime \prime}$ für Konzentrationen von $10^{20} \mathrm{Di}$ polen pro $\mathrm{cm}^{3}$ Lösung. Interpolationskurven nach Fröhlich Gl. (6). Analysedaten in Tab. 2.

Tab. 2. Analyse nach Fröhlich Gl. (6) der Absorptionsmessungen zur Temperaturabhängigkeit der Verteilungsbreite - (Abb. 5.1 und 5.2) - Dispersionsstufe $S$ für $10^{20}$ Dipole pro $\mathrm{cm}^{3}$ Lösung.

ten entsprechend einem $p=2,4 \quad\left(20{ }^{\circ} \mathrm{C}\right), \quad p=2,2$ $\left(40^{\circ} \mathrm{C}\right)$ und $p=2,0\left(60^{\circ} \mathrm{C}\right)$ mit Nujol als Lösungsmittel zugrunde liegen. Analog erhält man für die Dekalinlösung: $p=1,8\left(20^{\circ} \mathrm{C}\right), p=1,6\left(40{ }^{\circ} \mathrm{C}\right)$ und $p=1,4\left(60^{\circ} \mathrm{C}\right)$. Auch die in der zitierten Arbeit ebenfalls untersuchten Lösungen zweier weiterer Naphthalinderivate - 1-Chlor- und 2-Methyl-substituierte -, die mit 1-Bromnaphthalin gut vergleichbar sind, zeigen ein gleichlaufendes Verhalten. Die angegebenen Cole-Parameter werden stets mit wach. sender Temperatur kleiner. Dasselbe gilt für d,lCampher in verdünnter Lösung nach Messungen von Kalman und Smyth ${ }^{13}$ sowie Vaughan, Purcell und Sмүтн ${ }^{17}$, deren Ergebnisse wir ebenfalls nach unse-

17 W. E. Vaughan, W. P. Purcell u. C. P. Sмyth, J. Am. Chem. Soc. 83, 571 [1961]. 
rer Methode analysieren können. Für Tetrachlorkohlenstoff als Lösungsmittel ist zwar in allen Fällen $\left(20{ }^{\circ} \mathrm{C}\right.$ bis $\left.60{ }^{\circ} \mathrm{C}\right) p$ kleiner als 0,7 und daher nicht genauer zu bestimmen, doch für Nujol als Lösungsmittel nimmt die Verteilungsbreite mit steigender Temperatur ebenfalls deutlich ab: $p=2,2\left(20^{\circ} \mathrm{C}\right)$, $p=2,0\left(40^{\circ} \mathrm{C}\right)$ und $p=1,8\left(60^{\circ} \mathrm{C}\right)$. Kalman und Smyth untersuchten außerdem noch weitere in Nujol gelöste starre Dipolmoleküle, wie 4-Brombiphenyl, Isoquinolin und Acridin. Die von ihnen angegebenen Cole-Parameter nehmen durchweg mit steigender Temperatur deutlich ab.

Erwähnt seien noch die Untersuchungen von HoLLAND, RoBERTs und Sмyth ${ }^{18}$ an 1,1,1-Trichloräthan als reiner Dipolsubstanz sowie in Tetrachlorkohlenstofflösung, und an 2,2-Dinitropropane, gelöst in Heptan und Nujol. Auch hier werden mit wachsender Temperatur abnehmende Werte $\alpha$ angegeben, wie auch von Curtis, McGeer, Rathmann und Sмутн ${ }^{19}$ für 1-Chlornaphthalin gelöst in Heptan und Paraffin. Doch ist in den beiden zuletzt zitierten Arbeiten wegen des schmalen Spektralbereiches, über den sich die Beobachtungen erstrecken, nur die qualitative Aussage zu verwerten, daß die Verteilungsbreite mit steigender Temperatur abnimmt. Die einzigen uns bekannten Untersuchungen, die ein dem bisher beschriebenen genau entgegengesetztes Verhalten der Verteilungsbreite aufzeigen, wurden von Zeil, Haase und Stiefvater ${ }^{2}$ veröffentlicht, die das Relaxationsverhalten des 1-Chlornaphthalins und 9-Bromphenanthrens in Schwefelkohlenstoff in Abhängigkeit von der Temperatur angeben.

\section{Diskussion}

Die Auswahl der untersuchten Dipolmoleküle und der verwendeten Lösungsmittel wurde wesentlich beeinflußt von der ursprünglichen Vermutung, daß die Breite einer zu beobachtenden Relaxationszeitverteilung vom Verhältnis der Molekülvolumina von $\mathrm{Di}$ pol- und Lösungsmittelmolekül abhängt. Je kleiner das Dipolmolekül im Vergleich zum Lösungsmittelmolekül ist, desto größer sollte der Verteilungsparameter $p$ sein. Unsere Meßergebnisse - Tab. 1 zeigen demgegenüber, daß die Verteilungsbreite in erster Linie nur vom Lösungsmittel abhängt, weit-

18 R. S. Holland, G. N. Roberts u. C. P. Sмyth, J. Am. Chem. Soc. 78, 20 [1956]. gehend unabhängig aber vom Dipolmolekül selbst ist - gleiche Temperatur vorausgesetzt.

In Tab. 1 sind die Lösungsmittel ihrer Viskosität nach geordnet. Es scheint eine gewisse Gesetzmäßigkeit zwischen der Breite der Relaxationszeiverteilung und der Viskosität zu bestehen, da eine Zunahme der Verteilungsbreite von Heptan $(\eta=0,42 \mathrm{cP}$ bei $\left.20^{\circ} \mathrm{C}\right)$ bis zur aliphatischen Mischung $(\eta=7,0 \mathrm{cP}$ bei $20^{\circ} \mathrm{C}$ ) festzustellen ist. Doch der recht auffallende Unterschied zwischen den $p$-Werten für Benzol und Methylcyclohexan mit nahezu gleicher Viskosität $(0,65 \mathrm{cP}$ bzw. $0,73 \mathrm{cP})$ zeigt schon, daß diese Zunahme nicht besonders gleichmäßig ist. Vergleicht man jedoch die $p$-Werte für Chlorcyclohexan in verschiedenen Lösungsmitteln und bei verschiedenen Temperaturen, aber für annähernd gleiche Viskosität miteinander, so findet man, daß $p=1,2$ für $\eta=0,65 \mathrm{cP}\left(\right.$ Benzol $\left.20^{\circ} \mathrm{C}\right), p=1,6$ für $\eta=0,695 \mathrm{cP}$ (Heptan $-20^{\circ} \mathrm{C}$ ), $p=1,6$ für $\eta=0,73 \mathrm{cP}$ (Methylcyclohexan $20^{\circ} \mathrm{C}$ ) und $p=0,7$ für $\eta=0,91$ (Dekalin $90^{\circ} \mathrm{C}$ ). Damit ist ganz deutlich gezeigt, daß die Viskosität allein nicht die Breite der Relaxationszeitverteilung bestimmt. Erhärtet wird diese Erkenntnis auch durch Ergebnisse anderer Autoren. So gibt beispielsweise ScHRöDER ${ }^{20}$ ein $p=0,8$ an für eine 5-proz. Lösung von 1-Bromnaphthalin in Tetrachlorkohlenstoff mit einer Viskosität von ca. $1,1 \mathrm{cP}$ bei $20^{\circ} \mathrm{C}$, während in Methylcyclohexan von uns ein $p=1,4$ beobachtet wird. Nach Messungen von GRubi und Sмyтн ${ }^{16}$ kommt demgegenüber für die verdünnte Lösung von 1-Bromnaphthalin in $\mathrm{Nu}$ jol $(\eta=211 \mathrm{cP})$ mit $p=2,4$ nur eine geringfügige Zunahme der Verteilungsbreite gegenüber der von uns untersuchten Lösung in der aliphatischen Mischung $(7 \mathrm{cP})$ mit $p=2,2$ in Frage.

\section{Verteilungsbreite und Platzwechseltheorie}

Betrachten wir die Temperaturabhängigkeit der Verteilungsbreite, wie sie an den als Beispiel gewählten Lösungen von Chlorcyclohexan in Dekalin und Heptan zu beobachten ist, so kann diese nach Abb. 6 im gegebenen Temperaturintervall durch

$$
p=a \cdot\left(1 / T-1 / T_{0}\right)
$$

beschrieben werden, mit zwei Konstanten $a$ und $T_{0}$, deren physikalische Bedeutung noch näher zu inter-

19 A. J. Curtis, P. L. McGeer, G. B. Rathmann u. C. P. Smyth, J. Am. Chem. Soc. 74, 644 [1952].

20 R. Schröder, Z. Naturforsch. 12 a, 731 [1957]. 
pretieren ist. Dazu seien die wesentlichen Züge der Überlegungen von $\mathrm{F}_{\text {RöHLICH }}{ }^{6}$ skizziert.

In der Flüssigkeit ist eine Richtungsänderung der Molekelachse beispielsweise eines Dipolmoleküls wegen der quasikristallinen Nahordnung nicht durch kontinuierliches Drehen möglich, sondern nur durch

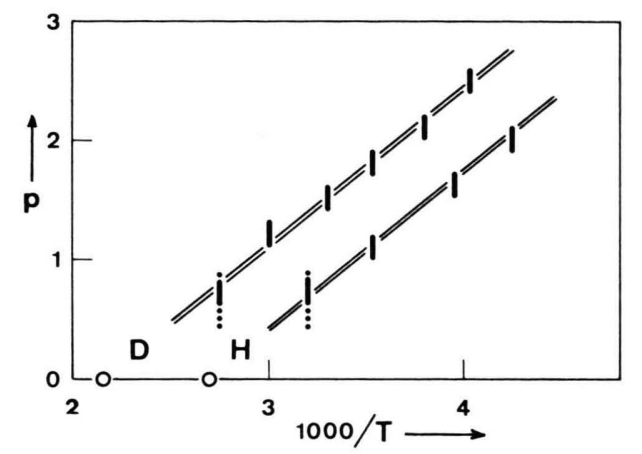

Abb. 6. Abhängigkeit der Breite der Relaxationszeitverteilung von der Temperatur. Aus den Messungen an verdünnten Lösungen von Chlorcyclohexan in Dekalin (D) und Heptan (H). Fröhlich-Parameter $p$ als Maß für die Breite der Verteilung.

sprunghafte Veränderung der Lage. Die Relaxationszeit als Maß für die Geschwindigkeit, mit der solche Lageänderungen stattfinden können, ist nach der Theorie der Platzwechselprozesse proportional zu den Übergangswahrscheinlichkeiten zwischen zwei benachbarten Gleichgewichtslagen, also

$$
1 / \tau \sim \exp (-H / k T) .
$$

$H$ ist hierbei die Höhe der Potentialwälle, die die einzelnen Gleichgewichtslagen trennen. Eine sich ständig ändernde Anordnung nächster Nachbarn in der Flüssigkeit wird sich nun darin äußern, daß die Höhe dieser Potentialwälle variiert. Das hat aber zur Folge, daß nicht eine einzige Relaxationszeit beobachtet werden wird, sondern ein $\tau$-Spektrum, dessen Verteilungsfunktion allerdings davon abhängt, wie die Verteilung der Potentialwallhöhen aussieht. Fröhlich setzt als einfachste Näherung eine Gleichverteilung über einen bestimmten Bereich an, so daß alle Höhen zwischen $H_{\mathrm{m}}-\frac{1}{2} b$ und $H_{\mathrm{m}}+\frac{1}{2} b$ gleich wahrscheinlich sind. Demnach sollten Relaxationszeiten auftreten zwischen $\tau_{1}$ und

$$
\tau_{2}=\tau_{1} \cdot \exp (-b / k T) .
$$

Diese Annahme führt zu der durch Gl. (3) und (4) gegebenen Relaxationszeitverteilung. Der Parameterwert ist proportional zur Variationsbreite der $\mathrm{Po}$ -

21 W. Kauzman, Rev. Mod. Phys. 14, 12 [1942]. tentialwallhöhen gemäß

$$
p=\ln \left(\tau_{1} / \tau_{2}\right)=b / k T .
$$

Mit anderen Annahmen über die Verteilung der Potentialwallhöhen könnten natürlich auch die übrigen angeführten Verteilungsfunktionen erhalten werden.

Nach Gl. (12) sollte $p$ direkt proportional zu 1/T sein und erst für $T$ gegen $\infty$ zu Null werden. Die Extrapolation der Geraden in Abb. 6 führt jedoch schon für endliche Temperaturen zu $p=0$, was mit unserem Ansatz (9) ausgedrückt worden ist. Diesen Ansatz können wir mit (12) verbinden zu

$$
p=V_{0}\left(1-T / T_{0}\right) / R T \text {. }
$$

Die Konstante $V_{0}$ ist ein Maß für die Variationsbreite der Potentialwallhöhen bzw. der auf ein Mol bezogenen Aktivierungsenergien; die Breite der Relaxationsverteilung wird zu Null beim Erreichen einer charakteristischen Temperatur $T_{0}$. Bemerkenswerterweise kann $T_{0}$ in beiden von uns beobachteten Fällen gleich der Siedetemperatur der jeweiligen Lösung gesetzt werden.

\section{Thermodynamische Aktivierungsgrößen}

Nach Kauzman ${ }^{21}$ und Eyring ${ }^{22}$ kann die Relaxationszeit im Sinne einer Theorie der absoluten Reaktionsgeschwindigkeiten durch thermodynamische Aktivierungsgrößen ausgedrückt werden :

$$
\tau=\tau_{0} \cdot \exp (\Delta G / R T) .
$$

Die Freie Enthalpie $\Delta G$ wird aufgeteilt in einen Anteil $\Delta U$, die Aktivierungsenergie, und $T \Delta S$, mit mit $\Delta S$ als Aktivierungsentropie, deren Mittelwerte aus der Temperaturabhängigkeit von $\tau_{\mathrm{m}}$ berechnet werden können, wenn $\tau_{\mathbf{0}}$ bekannt ist.

$$
\Delta G_{\mathrm{m}}=\Delta U_{\mathrm{m}}-T \Delta S_{\mathrm{m}} .
$$

Das Auftreten von Relaxationszeitverteilungen kann nun so gedeutet werden, daß Schwankungen dieser Aktivierungsgrößen um den Mittelwert auftreten, analog zu den Schwankungen der Potentialwallhöhen um ihren Mittelwert $H_{\mathrm{m}}$. Der Variationsbreite $b$ dieser Potentialwallhöhen entspricht dann eine Variationsbreite $V$ der Freien Enthalpie. Statt $H_{\mathrm{m}} \pm \frac{1}{2} b$ kann im Einklang mit Gl. (15) und (13)

$$
\Delta G_{\mathrm{m}} \pm \frac{1}{2} V=\left(\Delta U_{\mathrm{m}} \pm \frac{1}{2} V_{0}\right)-\left(\Delta S_{\mathrm{m}} \pm \frac{1}{2} V_{0} / T_{0}\right) \cdot T
$$

\footnotetext{
22 S. Glasstone, K. J. Laidler u. H. Eyring, Theory of Rate Processes, McGraw-Hill, New York 1941.
} 
geschrieben werden. Will man Zahlenwerte für $\Delta U_{\mathrm{m}}$ und $\Delta S_{\mathrm{m}}$ aus unseren Meßergebnissen berechnen, so muß man beachten, daß diese entscheidend davon abhängen, welcher Ansatz für $\tau_{0}$ gemacht wird. Kauzman setzt $\tau_{0}=h / k T$. Experimentelle Ergebnisse von Hufnagel ${ }^{23}$ lassen aber in den von uns betrachteten Fällen eher ein Anwachsen von $\tau_{0}$ mit der Temperatur erkennen. Zur Bestimmung von $\Delta U_{\mathrm{m}}$ kann schließlich auch einfach $\tau_{0}=$ const gesetzt werden. Die unter diesen Bedingungen erhaltenen Aktivierungsgrößen und ihre Variationsbreiten, die natürlich unabhängig von den eben erwähnten Schwierigkeiten einfach aus Abb. 6 entsprechend Gl. (13) gewonnen werden können, sind in Tab. 3 zusammengestellt.

\begin{tabular}{|c|c|c|c|c|}
\hline \multirow[b]{2}{*}{ Lösungsmittel } & \multicolumn{3}{|c|}{$\Delta U[\mathrm{kcal} / \mathrm{Mol}]$} & \multirow{2}{*}{$\frac{V_{0}}{2}$} \\
\hline & Kauzman & $\tau_{0}=$ const & Hufnagel & \\
\hline Heptan & 1,2 & 1,6 & 2,3 & 1,3 \\
\hline \multirow[t]{3}{*}{ Dekalin } & 2,0 & 2,5 & 3,4 & 1,3 \\
\hline & \multicolumn{3}{|c|}{$\Delta S[\mathrm{cal} / \mathrm{MolGrd}]$} & $V_{0}$ \\
\hline & Kauzma & & UFNAGEL & $2 T_{0}$ \\
\hline Heptan & $-2,6$ & & 0,9 & 3,5 \\
\hline Dekalin & $-1,6$ & & 1,9 & 2,8 \\
\hline
\end{tabular}

Tab. 3. Chlorcyclohexan in verdünnter Lösung - Mittlere Aktivierungsenergie $\Delta U$ und -entropie $\Delta S$, berechnet aus der Temperaturabhängigkeit der mittleren Relaxationszeit $\tau_{\mathrm{m}}$, sowie die Variationsbreite der Aktivierungsgrößen, berechnet aus der Temperaturabhängigkeit des Verteilungsparameters $p$ (Tab. 2).

\section{Verallgemeinerung}

Die Ergebnisse in Tab. 3 ermutigen uns, auch für die Lösungen des Chlorcyclohexans in den anderen Lösungsmitteln als charakteristische Temperatur $T_{0}$ die Siedetemperatur anzusetzen. Dann kann die entsprechende Variationsbreite $V_{0}$ aus den $p$ Werten für $20{ }^{\circ} \mathrm{C}$ berechnet werden. Für Chlorcyclohexan als Dipolmolekül erhalten wir in Benzol $V_{0}=3,9$, in Methylcyclohexan $V_{0}=4,2$ und in der aliphatischen Mischung $V_{0}=4,5$ gegenüber $V_{0}=2,6$ für Heptan und Dekalin als Lösungsmittel (Dimension von $\left.V_{0}:[\mathrm{kcal} / \mathrm{Mol}]\right)$. Die Variationsbreite der Potentialwallhöhen bzw. der Aktivierungsenergien ändert sich beim Wechsel der Lösungsmittel somit höchstens um einen Faktor 2.

Erweitern wir diese Verallgemeinerung auf alle untersuchten Dipolsubstanzen - mit Ausnahme des Tetrahydrofurans, für das die $p$-Werte nur angenommen wurden - , so sind die resultierenden Varia-

23 F. Hufnagel, Habilitationsschrift, Mainz 1967. tionsbreiten genauso einheitlich wie die zur Berechnung herangezogenen Parameterwerte $p$ von Tab. 1, wie in Tab. $4 \mathrm{zu}$ erkennen ist. $\mathrm{Zu}$ beachten sind die unterschiedlichen Fehlergrenzen, die natürlich direkt aus der unterschiedlichen Genauigkeit kleiner und großer $p$-Werte folgen.

\begin{tabular}{|c|c|c|c|c|c|}
\hline \multirow{2}{*}{$\begin{array}{c}\text { D i pol- } \\
\text { mole k ü l } \\
\text { Benzophenon }\end{array}$} & Heptan & \multicolumn{3}{|c|}{ Lösungsmit tel } & $\begin{array}{l}\text { aliph. } \\
\text { Mischg. }\end{array}$ \\
\hline & 1,9 & 3,3 & 3,7 & $(2,5)$ & $(3,4)$ \\
\hline 1-Bromnaphthalin & & 3,3 & 3,7 & 2,8 & 4,2 \\
\hline Chlorcyclohexan & 2.6 & 3,9 & 4,2 & 2,6 & 4,5 \\
\hline Chlorbenzol & 1,9 & 3,3 & 3,7 & 2,5 & 4,2 \\
\hline Fehlergrenzen \pm & 0,8 & 0,3 & 0,3 & 0,2 & 0,2 \\
\hline$T_{0}\left[{ }^{\circ} \mathrm{K}\right]$ (Siedetemp.) & 371 & 353 & 373 & 463 & 423 \\
\hline
\end{tabular}

Tab. 4. Variationsbreite der Aktivierungsenergie in kcal/Mol, berechnet aus den Verteilungsparametern $p$ (Tab. 1) für $20^{\circ} \mathrm{C}$ unter der Annahme $T_{0}=$ Siedetemperatur der Lösung. Werte für Chlorcyclohexan in Heptan und Dekalin nach Abb. 6 und Gl. (13).

Interessant ist noch ein Weiterführen dieser Verallgemeinerung auf stärker konzentrierte Lösungen. SCHRöDER ${ }^{20}$ berichtet über eine mit der Konzentration zunehmende Verbreiterung der Absorptionskurven von 1-Bromnaphthalin in Tetrachlorkohlenstoff. Da auch die Siedetemperatur mit der Konzentration anwächst, könnte unserem Ansatz zufolge selbst dann, wenn $V_{0}$ als weitgehend unabhängig von der Konzentration angenommen wird, eine Änderung von $T_{0}$ gleichsinnig mit der Siedetemperatur die Zunahme der Verteilungsbreite bewirken. Aus den Zahlen, die Schröder mitteilt, kann für die 20-proz. Lösung mit $p=1,2$ ein $V_{0}=3,5 \mathrm{kcal} / \mathrm{Mol}$ abgeschätzt werden. Geringer konzentrierte Lösungen, deren $T_{0}$ gleich der Siedetemperatur zu setzen wäre, dürften bei gleichem $V_{0}$ nur ein $p$ von etwa 0,9 zeigen, wie es tatsächlich der Fall ist. Um sicherere Aussagen zu gewinnen, bedarf es freilich wesentlich umfangreicherer Meßergebnisse.

\section{Schluß}

Die Ergebnisse unserer Untersuchungen führen uns zu folgendem Schluß: Die Breite einer Relaxationszeitverteilung, wie sie für starre Dipolmoleküle in verdünnter Lösung zu beobachten ist, wiedergegeben durch einen Parameter $p$, wird durch eine für die Lösung charakteristische Temperatur $T_{0}$ - möglicherweise mit der Siedetemperatur verknüpft - und die Schwankungsbreite $V_{0}$ der Potentialwallhöhen bzw. der Aktivierungsenergien be- 
stimmt. Der Parameter $p$, der nach $p=\ln \left(\tau_{1} / \tau_{2}\right)$ mit der Breite der Verteilung - gegeben durch zwei "Grenzen “ $\tau_{1}$ und $\tau_{2}$ - zusammenhängt, zeigt eine Temperaturabhängigkeit, die durch

$$
p=V_{0}\left(1-T / T_{0}\right) / R T
$$

beschrieben werden kann. Mittlere Aktivierungsenergie und Schwankungsbreite haben dieselbe Größenordnung von einigen wenigen kcal/Mol.
Herrn Prof. Dr. G. Klages danke ich herzlichst für die Anregung zu dieser Arbeit sowie für viele fruchtbringende Diskussionen. Ebenso danke ich den Mitarbeitern in der Abteilung für Mikrowellenphysik für ihre kollegiale Hilfe, besonders Herrn Dozent Dr. F. Hufnagel, sowie den Herren G. Krauss, D. Grätzer und H. KILP für die Durchführung der Messungen mit 33 bis $11 \mathrm{~cm}-, 4$ und 2,3 mm-Wellen. Die Deutsche Forschungsgemeinschaft ermöglichte die Untersuchungen durch ihre großzügige Unterstützung.

\title{
ENDOR-Untersuchungen am metastabilen Triplett-Zustand des Naphthalin-Moleküls
}

\author{
P. Ehret und H. C. Wolf \\ 3. Physikalisches Institut der Universität Stuttgart \\ (Z. Naturforsch. 23 a, 1740-1746 [1968]; eingegangen am 17. August 1968)
}

\begin{abstract}
ENDOR-measurements of the photo-excited triplet state of naphthalene, 1-monodeutero-naphthalene and 1,4-dideutero-naphthalene in durene at $4.2{ }^{\circ} \mathrm{K}$ are reported. The hyperfine interaction constants of $\alpha$ - and $\beta$-protons and $\alpha$-deuterons, the corresponding spin densities and the axis of the hyperfine tensor are determined. A doublet splitting of all ENDOR lines indicates the existence of four (instead of two) inequivalent sites for naphthalene molecules in the durene matrix.
\end{abstract}

Die Elektronenspinresonanz (ESR) des metastabilen Triplett-Zustandes organischer Moleküle wurde erstmals am Naphthalin in einer einkristallinen Durol-Matrix (Tetramethylbenzol) nachgewiesen ${ }^{1}$. Man beobachtet eine Feinstruktur wegen der Dipol-DipolKopplung der beiden Elektronen im Triplett-Zustand und zusätzlich eine teilweise aufgelöste Hyperfeinstruktur (Hfs) wegen der magnetischen ElektronProton-Wechselwirkung. Die sorgfältige ESR-Analyse dieser $\mathrm{Hfs}$ in verschieden deuterierten Naphthalin-Molekülen ermöglicht die Berechnung eines Teils der Wechselwirkungskonstanten ${ }^{2}$ mit den $\alpha$ - und $\beta$ Protonen. Aus einer ENDOR-Untersuchung erhofft man sich die lückenlose Messung aller Hfs-Konstanten der $\alpha$ - und $\beta$-Protonen mit erhöhter Genauigkeit und die Bestimmung der Hauptachsen des Hfs-Tensors aus der Winkelabhängigkeit der ENDOR-Spektren. Eine ausführliche ENDOR-Untersuchung an einem aromatischen Molekül im Triplett-Zustand

1 C. A. Hutchison jR. u. B. W. Mangum, J. Chem. Phys. 34, 908 [1961].

2 N. Hirota, C. A. Hutchison jr. u. P. Palmer, J. Chem. Phys. 40, 3717 [1964].

3 C. A. Hutchison jr. u. G. A. Pearson, J. Chem. Phys. 43, 2545 [1965]. wurde bisher nur für den Fall eines Triplett-Grundzustandes durchgeführt ${ }^{3,4}$.

In der vorliegenden Arbeit wird am optisch angeregten Triplett-Zustand des Naphthalin-Moleküls die ENDOR-Methode zur genauen Analyse der Hyperfeinwechselwirkung mit den Protonen benutzt. Dazu wurden folgende Moleküle in eine Durol-Matrix eingebaut: Naphthalin- $\mathrm{h}_{8}\left(\mathrm{~N}-\mathrm{h}_{8}\right), \mathrm{l}-\mathrm{Monodeuteronaph-}$ thalin $\left(\mathrm{N}-\mathrm{d}_{1}\right)$ und 1,4-Dideuteronaphthalin $\left(\mathrm{N}-\mathrm{d}_{2}\right)$.
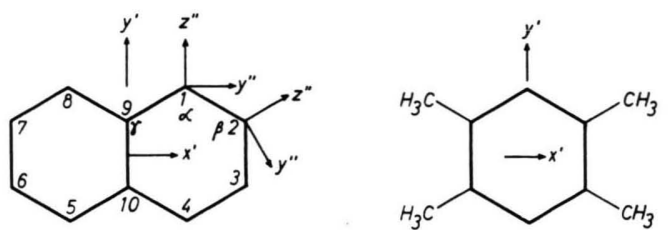

Abb. 1. Achsensysteme der Naphthalin- und Durol-Moleküle. $x^{\prime}, y^{\prime}, z^{\prime}=$ Feinstruktur-Hauptachsensystem; $x^{\prime \prime}, y^{\prime \prime}, z^{\prime \prime}=$ Hyperfeinstruktur-Hauptachsensystem ( $z^{\prime \prime}$ in $\mathrm{C}-\mathrm{H}$-Richtung).

Erste Ergebnisse aus der vorliegenden Arbeit wurden bereits veröffentlicht ${ }^{5}$.

${ }^{4}$ C. A. Hutchison jr. u. G. A. Pearson, J. Chem. Phys. 47, 520 [1967].

5 P. Ehret, G. Jesse u. H. C. Wolf, Z. Naturforsch. 23 a, 195 [1968].

6 J. M. Robertson, Proc. Roy. Soc. London A 141, 594 [1933]; A 142, 659 [1933]. 